\title{
ASSOCIAÇÃO DO ÍNDICE DE MASSA CORPORAL, FORÇA MUSCULAR E NÍVEL DE ATIVIDADE FÍSICA DE IDOSAS PRATICANTES DE HIDROGINÁSTICA
}

\author{
Daniel Vicentini de OLIVEIRA ${ }^{1}$ \\ Mateus Dias ANTUNES ${ }^{2}$ \\ Denner Júnior LEITE ${ }^{3}$ \\ Daniel Eduardo da Cunha LEME
}

José Roberto Andrade do NASCIMENTO JÚNIOR ${ }^{5}$

\begin{abstract}
${ }^{1}$ Doutorando em Gerontologia pela Universidade Estadual de Campinas (UNICAMP) - d.vicentini@ hotmail.com ${ }^{2}$ Mestrando em Promoção da Saúde pelo Centro Universitário de Maringá (UNICESUMAR) - Bolsista Capes, mateus_antunes03@hotmail.com ${ }^{3}$ Graduado em Educação física pela Faculdade Metropolitana de Maringá (FAMMA) -dennerjunior87@gmail.com ${ }^{4}$ Mestrando em Gerontologia pelaUniversidade Estadual de Campinas (UNICAMP) -daniel.eduardo.7@ hotmail.com ${ }^{5}$ Doutor em Educação física pela Universidade Estadual de Maringá (UEM) - jroberto.jrs01 @ gmail.com.
\end{abstract}

Recebido em: 03/10/2016 - Aprovado em: 09/01/2017 - Disponibilizado em: 01/07/2017

\begin{abstract}
RESUMO:
O objetivo do presente estudo foi analisar a associação do Índice de Massa Corporal (IMC), força muscular (FM) e o nível de atividade física (NAF) de idosas praticantes de hidroginástica. Metodologia: estudo transversal, realizado com 110 idosas com idade entre 60 e 80 anos, praticantes de hidroginástica. As participantes foram avaliadas em relação ao peso corporal, IMC, FM em membros superiores por meio do Teste de flexão de cotovelo, e membros inferiores pelo Teste Levantar e Sentar na cadeira em 30 segundos, além de variáveis demográficas e socioeconômicas. O NAF foi mensurado por meio do Questionário Internacional de Atividade Física (IPAQ), versão curta. Resultados: do total de idosas avaliadas, 29,1\% foram classificadas com FM fraca de membros inferiores. A maioria $(77,3 \%)$ foi considerada ativa ou muito ativa em relação ao NAF. O IMC $(\mathrm{p}=0,002)$ e a FM de membro inferior $(\mathrm{p}=0,007)$ associaram-se ao $\mathrm{NAF}$, sendo que nas idosas ativas o IMC foi elevado, enquanto que o nível de FM em membros inferiores foi fraco/muito fraco nas idosas ativas ou muito ativas. Conclusão: Existe associação inversa do NAF com a FM de membros inferiores de idosaspraticantes de hidroginástica, indicando que idosas ativas e muito ativas possuem FM fraca/muito fraca.
\end{abstract}

Palavras-Chave: Atividade Física. Envelhecimento. Promoção da Saúde.

\begin{abstract}
:
The aim of this study was to analyze the association of body mass index (BMI), muscle strength (MS) and the level of physical activity (LPA) of older adult women engaged in hydrogymnastics. Methodology: Cross-sectional study with 110 older adult women, aged 60 and 80, hydrogymnasticspractitioners. Participants were evaluated for body weight, BMI, MS in upper limbs through the elbow flexion test, and lower limbs by test Lift and Sit in the chair in 30 seconds, as well as demographic and socioeconomic variables. The NAF was measured using the International Physical Activity Questionnaire (IPAQ), short version. Results: of the total older adult women, 29.1\% were classified as weak LS lower limbs. The majority (77.3\%) were considered active or highly active compared to the NAF. BMI $(p=0.002)$ and LS lower limb $(\mathrm{p}=0.007)$ were associated with the NAF, and the active elderly BMI was high, while the FM level in the lower limbs was poor / very poor in elderly active or very active. Conclusion: There is an inverse association with NAF FM lower limbs of elderly women engaged in aerobics, indicating that active and very active women have weak FM / very poor
\end{abstract}

Keywords: Physical activity. Aging. Health Promotion 


\section{INTRODUÇÃO}

O envelhecimento é um processo dinâmico, progressivo, universal e individual, caracterizado por alterações físicas, funcionais e psicológicas (SILVA; DAL PRÁ, 2014). As modificações corporais tais como a redução da massa corporal magra e o acúmulo de gordura visceral são comuns com o avançar da idade e associam-se ao desenvolvimento de doenças crônicas e incapacidade funcional nas Atividades Básicas de Vida Diária (ABVD) e Atividades Instrumentais de Vida Diária (AIVD) (KÜMPEL et al., 2011).

Entre 25 e 65 anos de idade, o ser humano perde de $10 \%$ a $16 \%$ da massa corporal magra, e até os 70 nos de idade a redução de fibras musculares do tipo II chega a 50\% (LEITE et al., 2012). Paralelamente à diminuição do número e do tamanho das fibras musculares, ocorre a redução acentuada da força muscular (FM), sendo uma perda de $1,5 \%$ a $5 \%$ ao ano (KELLER; ENGELHARDT, 2013).

Os exercícios físicos regulares auxiliam na redução do peso corporal, no ganho de FM, na manutenção da capacidade funcional e autonomia, contribuindo para a melhora da saúde e qualidade de vida da pessoa idosa. A hidroginástica possui a vantagem do menor impacto nas articulações, devido às propriedades físicas da água: flutuação, resistência e pressão hidrostática, sendo uma modalidade apropriada para os idosos, visto que muitos deles apresentam doenças osteoarticulares (SOUTO et al., 2016).

Mediante os benefícios da prática do exercício físico, especificamente a hidroginástica na saúde do idoso, o presente estudo teve por objetivo analisar a associação entre o IMC, FM e o nível de atividade física (NAF) de idosas praticantes de hidroginástica das academias do município de MaringáParaná.

\section{MATERIAIS E MÉTODOS}

Trata-se de um estudo descritivo, quantitativo, transversal e observacional. A amostra foi composta de idosas entre 60 e 80 anos de idade, praticantes exclusivamente de hidroginástica há pelo menos três meses e frequência de duas vezes na semana em nove academias do município de Maringá, estado do Paraná.

Foram excluídos os idosos do sexo masculino, devido à baixa prevalência dos mesmos na prática de hidroginástica, assim como as idosas que praticassem outras modalidades de exercício físico; que utilizassem prótese de joelho e quadril; submetidas à cirurgia de joelho e quadril nos últimos seis meses; que utilizassem dispositivos de auxílio para marcha; com déficits importantes na audição e visão que impedissem a realização das avaliações 
durante a coleta de dados. A amostra foi selecionada de forma intencional por conveniência, sendo composta por 110 idosas.

Para caracterização da amostra foi utilizado um questionário sócio demográfico composto por 14 questões referentes à idade, raça, escolaridade, tabagismo, renda mensal (SM = salário mínimo), situação conjugal, trabalho remunerado atual, aposentadoria, auto percepção de saúde, identificação de atividade física como benefício para a saúde e prescrição de atividade física no último ano.

O peso corporal foi avaliado por meio da balança digital QF - 2003®, no qual o avaliado permanecia descalço, com o corpo ereto e olhando para frente (horizonte) para a referida medida. A estatura foi avaliada com estadiômetro da marca SIM®. Solicitou-se que a idosa ficasse ao lado do mesmo, descalça, para verificação da altura. A partir dos dados do peso corporal e altura, foi calculado o IMC com a fórmula IMC $=$ Peso $(\mathrm{Kg}) /($ Altura(m))2. Resultados abaixo de 18,5 foram considerados como desnutrição; entre 18,5 e 24,5 peso normal; entre 25,0 e 29,0 sobrepeso; entre 30,0 e 39,0 obesidade e acima de 40,0 obesidade mórbida (CERVI; FRANCESCHINI; PRIORE, 2005).

A FM de membros inferiores foi avaliada por meio do "Teste levantar e sentar na cadeira", em 30 segundos. Para tanto, os participantes deveriam cruzar os braços junto ao tórax, efetuando o movimento de sentar e levantar sem auxílio das mãos, utilizando apenas a força dos membros inferiores. Foram coletados os números de repetições (levantar e sentar) em um tempo de 30 segundos (RIKLI, 2008). Para avaliação da FM dos membros superiores, aplicou-se o "Teste de flexão de cotovelo" do membro superior dominante, com halter de dois quilos durante o tempo de 30 segundos. Foram registradas as flexões de cotovelo efetuadas durante o período mencionado (SANTOS et al., 2013).

O NAF foi mensurado por meio do IPAQ - Questionário Internacional de Atividade Física, versão curta do CELAFISCS - Centro de Estudos do Laboratório de Aptidão Física de São Caetano do Sul (2016), composto por 4 questões. Neste instrumento, o participante deve relatar as atividades físicas realizadas em um tempo mínimo de 10 minutos contínuos, nas últimas duas semanas em casa, trabalho e no transporte, enquanto exercício ou lazer. Além disso, foi feito o registro do tempo de caminhada nos dias em que tais atividades foram praticadas. Posteriormente os participantes relataram a quantidade de dias da semana nos quais realizaram atividades moderadas em um tempo mínimo de 10 minutos contínuos, dentre elas andar de bicicleta, nadar, dançar, limpar a casa entre outras com exceção da caminhada. Em seguida foi questionado o 
tempo total gasto nas atividades realizadas por dia. Por último, foi registrada a prática de atividades físicas vigorosas, durante os dias da última semana. O tempo total gasto na prática de tais atividades por dia foi registrado. Por último, as idosas foram questionadas em relação ao tempo total gasto na posição sentada, durante um dia da semana e em um dia de final de semana (MATSUDO et al., 2001).

As coletas de dados ocorreram no período de Junho a Agosto do ano de 2016, após autorização das academias do município de Maringá - PR. As idosas praticantes de hidroginástica foram informadas quanto à justificativa e objetivo do trabalho, e aquelas que aceitaram participar da pesquisa assinaram o Termo de Consentimento Livre e Esclarecido (TCLE).

Foram utilizadas a frequência e percentual para as variáveis categóricas e para as variáveis numéricas, inicialmente foi verificada a normalidade dos dados por meio do teste de Kolmogorov-Smirnov, no qual mostrou que a distribuição não foi normal, sendo assim foram utilizadas Medianas (Md) e Quartis (Q1; Q3) para a caracterização dos resultados. Na comparação do IMC, FM de membros inferiores e superiores em função do NAF (Sedentário/Irregularmente ativo, ativo, muito ativo), foi utilizado o teste de Kruskal-Wallis seguido do teste "U" de Mann-Whitney para pares de grupos. O teste de Qui-quadrado de Pearson (X2) foi utilizado para observar as possíveis associações existentes da FM e IMC com o NAF. Considerou-se um nível de significância de $\mathrm{p}<0,05$.

Este estudo foi aprovado pelo Comitê de ética em Pesquisa com seres humanos (CEP) do Centro Universitário Cesumar (UNICESUMaR) por meio do parecer número 1.745.616/2016.

\section{RESULTADOS}

A amostra foi composta predominantemente por idosas com idade até 70 anos (64,5\%), casadas $(57,3 \%)$ e caucasianas $(93,6 \%)$. Em relação ao perfil socioeconômico a maioria $(41,8 \%)$ possuía o ensino médio completo, renda mensal de três salários mínimos $(56,4 \%)$ e apesar de aposentada $(86,4 \%)$ possuía trabalho remunerado $(91,8 \%)$. A prevalência de idosas que nunca fumaram foi de $69,1 \%$.

Com relação ao perfil de saúde e atividade física das, verificou-se que a maioria possuía percepção de saúde boa $(47,3 \%)$ e apresentou eutrofia $(53,6 \%)$. Com relação à FM de membros inferiores, 29,1\% obteve classificação fraca no teste sentar e levantar. Quanto à FM de membro superior dominante no teste de flexão de cotovelo, $34,5 \%$ obteve nível de força muito bom. No que se refere ao NAF, $77,3 \%$ das idosas apresentaram nível ativo ou muito ativo. 
Houve diferença significativa no IMC $(\mathrm{p}=0,002)$ e no teste de sentar e levantar ( $\mathrm{p}$ $=0,007)$, indicando que as idosas ativas apresentaram maior IMC quando comparadas com as muito ativas e sedentárias/irregularmente ativas, que por sua vez, apresentaram maior escore no teste de sentar e levantar em detrimento as demais idosas (Tabela 1). Por último, verificou-se associação significativa do NAF com o teste de sentar e levantar $(\mathrm{p}=0,006)$. As idosas ativas ( $\mathrm{f}=22$ ) e muito ativas ( $\mathrm{f}=22$ ) obtiveram o nível fraco/muito fraco no teste de sentar e levantar (Tabela 2).

Tabela 1- Distribuição da frequência do perfil de saúde e atividade física em idosas praticantes de hidroginástica do município de Maringá-PR.

Nível de Atividade Física

\begin{tabular}{|c|c|c|c|c|}
\hline \multirow{2}{*}{ Variáveis } & $\begin{array}{l}\text { 1.Sedentário/Irreg. Ativo } \\
(\mathbf{n}=\mathbf{2 5})\end{array}$ & $\begin{array}{l}\text { 2.Ativo } \\
(\mathbf{n}=\mathbf{5 0})\end{array}$ & $\begin{array}{l}\text { 3.Muito Ativo } \\
\quad(n=35)\end{array}$ & \multirow[b]{2}{*}{$\boldsymbol{P}$} \\
\hline & Md (Q1;Q3) & Md (Q1;Q3) & Md (Q1;Q3) & \\
\hline Idade (anos) & $65,0(63,5 ; 69,0)$ & $67,5(62,7 ; 71,0)$ & $68,0(64,0 ; 73,0)$ & 0,566 \\
\hline Peso (kg) & $71,0(64,0 ; 75,0)$ & $72,8(65,7 ; 76,2)$ & $69,0(63,0 ; 75,0)$ & 0,071 \\
\hline Estatura (m) & $1,61(1,57 ; 1,69)$ & $160,5(1,58 ; 1,67)$ & $1,63(1,58 ; 1,67)$ & 0,805 \\
\hline $\operatorname{IMC}\left(\mathrm{kg} / \mathrm{m}^{2}\right)$ & $26,0(25,2 ; 27,3)$ & $27,5(26,0 ; 29,0)^{\mathrm{a}}$ & $25,4(24,0 ; 27,7)$ & $0,002 *$ \\
\hline FM de membro inferior & $16,0(14,0 ; 19,0)^{\mathrm{b}}$ & $15,5(12,0 ; 17,0)$ & $14,0(12,0 ; 16,0)$ & $0,007 *$ \\
\hline FM de membro superior & $19,0(16,0 ; 21,00$ & $19,0(16,7 ; 22,0)$ & $20,0(17,0 ; 22,0)$ & 0,228 \\
\hline
\end{tabular}

* Diferença significativa: $\mathrm{p}<0,05$ - Teste de Kruskal-Wallis

Irreg.: irregularmente; Kg: quilogramas; m: metros; FM: força muscular.

Tabela 2 - Associação do nível de atividade física com o IMC e força muscular das idosas praticantes de hidroginástica do município de Maringá-PR.

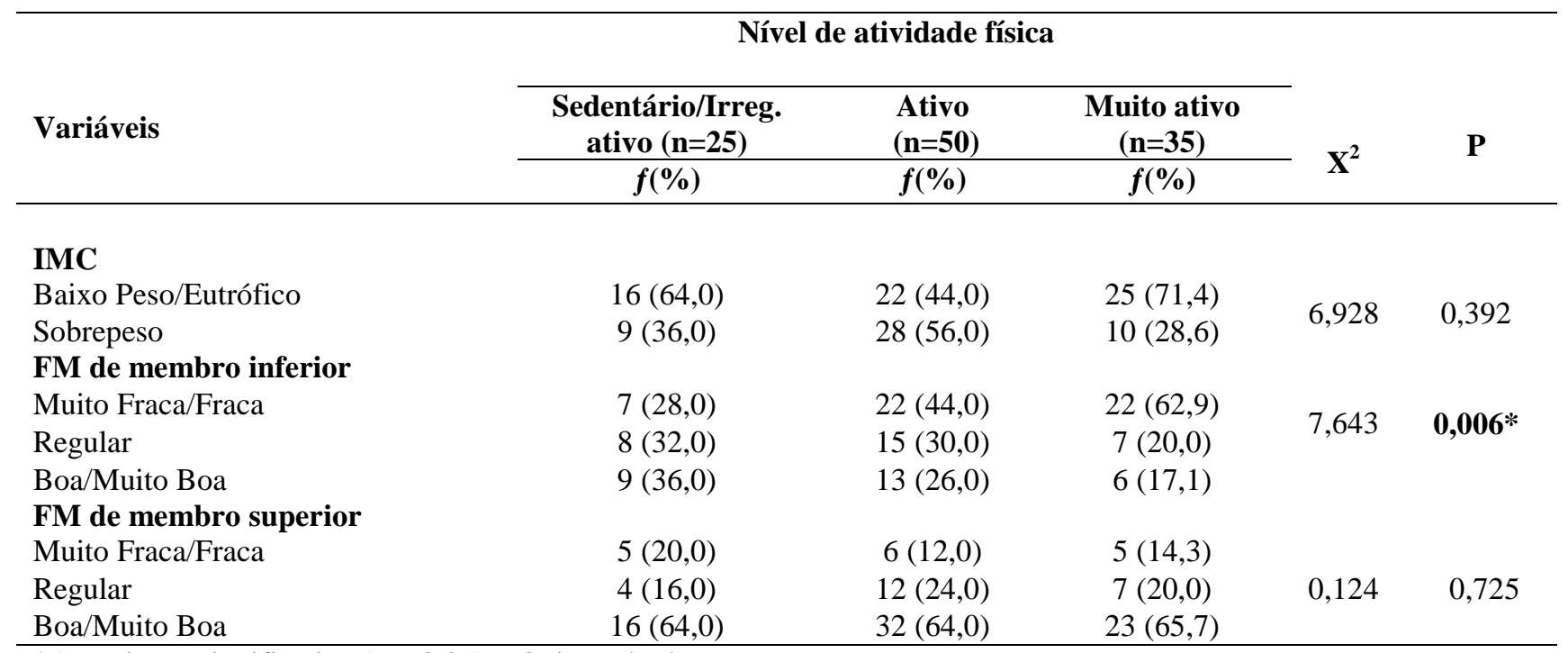

*Associação significativa ( $<$ 0,05) - Qui-quadrado.

Irreg: irregularmente ativo; IMC: Índice de massa corporal; FM: força muscular. 


\section{DISCUSSÃO}

O IMC foi maior entre as pessoas idosas ativas, contudo não significa relação com o sobrepeso nas participantes da pesquisa. A literatura ressalta que na velhice as mulheres possuem um nível elevado de IMC. Segundo Sgariboldi et al. (2015), as características antropométricas e de distribuição da gordura corporal entre as idosas são influenciadas pela idade. No entanto, os autores ressaltam que embora que o IMC seja um dos meios de caracterização e/ou definiçãa do excesso de peso corporal, não descartam o uso da bioimpedância para este fim.

A utilização do IMC e limites de normalidade nas análises de desnutrição, obesidade e sobrepeso entre a população idosa, vem sendo amplamente discutida, pois nesta faixa etária há um decréscimo acentuado da estatura, acúmulo do tecido adiposo, redução da massa corporal magra e diminuição da quantidade de água no organismo, devido às perdas do envelhecimento e tais alterações dificultam o emprego deste instrumento, mesmo se tratando do indicador antropométrico mais utilizado, de fácil aplicação, baixo custo e não invasivo (SOUZA et al., 2013).

Apesar da variabilidade nos índices de IMC entre as participantes da pesquisa, a prática da hidroginástica é relevante para os idosos. Tal modalidade contribui para a manutenção do nível saudável de gordura corporal. Em um estudo recente Vieira (2014), aponta a hidroginástica como um exercício físico eficiente na redução da gordura, uma vez que durante a realização desta atividade há produção de massagens em regiões corporais que estão em movimento, além da queima de calorias devido os exercícios aeróbicos e anaeróbicos realizados na água, refletindo nos níveis adequados de IMC.

Por outro lado, Nesi(2015) observou que nas idosas acima do peso praticantes de hidroginástica, a queima de calorias mediante o referido exercício somado ao acompanhamento nutricional, proporciona a perda de peso corporal. Além disso, para as idosas em níveis adequados de IMC foi observado que a atividade física evita o ganho de peso. Portanto, concluíram que a manutenção de um IMC adequado auxilia na imagem corporal e prevenção de doenças crônicas e incapacitantes, sendo fatores fortemente associados às melhores condições de saúde.

Zucolo et al.(2016) investigaram os efeitos da hidroginástica sobre variáveis morfológicas e funcionais de pessoas de meia idade e idosos por meio da massa corporal, IMC, relação cintura-estatura, flexibilidade de cadeia posterior e FM de membros superiores e inferiores. Foi observado diminuição significativa da massa corporal e aumento significativo no desempenho dos 
testes de FM de membros superiores e inferiores, já as demais variáveis não foram encontradas alterações significativas. Concluindo que a intervenção com a hidroginástica promove efeitos positivos em alguns aspectos morfológicos e funcionais em pessoas de meia idade e idosos.

Kura et al. (2006) verificaram o NAF, o IMC e a FM estática entre idosas praticantes de ginástica e hidroginástica pertencentes ao Centro Regional de Estudos e Atividade para Terceira Idade. Ambos os grupos apresentaram predominância por mulheres ativas com baixo nível de FM de membros inferiores. Quanto ao IMC, observou-se que os grupos estavam dentro dos padrões apresentados pela literatura. Assim, as atividades desempenhadas pela amostra do estudo tem um papel importante no comportamento para as variáveis estudadas, no entanto, ainda deve incrementar na rotina das idosas, exercícios que desenvolvam a FM a fim de estabilizar ou aumentar tais índices.

Vedana et al. (2011) ao analisarem a influência da hidroginástica sobre a composição corporal, aspectos cardiovasculares, hematológicos, função pulmonar e aptidão física de adultos e idosos durante duas semanas antes do início da hidroginástica e após 16 semanas de intervenção, encontraram uma significante redução no IMC, adiposidade corporal, endomorfismo, frequência cardíaca de repouso, aumento na massa muscular magra, $\mathrm{SpO} 2$ e hematócrito, melhora das capacidades pulmonares e da flexibilidade, mas não de FM.

No estudo de Costa, Carvalho e Gomes (2016) ao verificarem o efeito das aulas de hidroginástica de um projeto de extensão, ao longo de 1 ano, nas capacidades físicas e em variáveis antropométricas dos idosos participantes 2 vezes por semana durante 1 ano conforme o calendário da Universidade houve a diminuição das variáveis antropométricas, dobras cutâneas e percentual de gordura, enquanto que a massa corporal e o IMC não mudaram. Em relação as capacidades físicas, a flexibilidade dos músculos isquiotibiais e dos posteriores de tronco melhoraram significativamente, porém a FM dos extensores do joelho e a potencia aeróbia não mudaram.

No estudo de Silva et al. (2012) que investigaram os efeitos de uma intervenção de curto prazo com a hidroginástica sobre indicadores de obesidade em idosas obesas frágeis, três vezes semanais por seis meses. Ao final da intervenção, houve tendência a melhorias sendo observada que as alterações mais importantes foram observadas durante o $2^{\circ}$ trimestres para todas as variáveis analisadas, sendo duas vezes maiores do que no $1^{\mathrm{o}}$ trimestre. Assim, as intervenções de curto prazo com a hidroginástica tende a produzir melhorias em indicadores de 
obesidade sendo uma alternativa viável para o tratamento da obesidade de idosos frágeis.

Com relação ao teste de sentar e levantar que avalia a FM de membros inferiores, a maioria das idosas ativas e muito ativas apresentou menores valores nos escores e foi classificada como fraca ou regular. Segundo Miranda et al.(2009), a perda muscular dos membros inferiores é comum e ocorre progressivamente com o avançar da idade, sendo um fator associado à perda da capacidade funcional e quedas. Apesar de a hidroginástica possuir benefícios para saúde como supramencionado, em qualquer das situações (idosas que pontuaram ou não desfavoravelmente no teste de FM de membros inferiores) há necessidade do exercício físico resistido específico a esta faixa etária, a fim de promover o aumento da massa, FM e flexibilidade muscular nos membros inferiores.

Entretanto, em nosso estudo não foram observados menores valores de FM em membros superiores entre as idosas avaliadas. Souza Júnior et al.(2015) ao avaliarem a FM de membros superiores e inferiores em idosas praticantes e não praticantes de ginástica funcional, notaram que as praticantes apresentaram maior força muscular em membros superiores com relação aos inferiores nos dois grupos avaliados. Comumente, as idosas utilizam os membros superiores na execução das atividades diárias, resultando em ganhos de força muscular neste segmento corporal comprado aos membros inferiores.

Vale ressaltar que as perdas nas propriedades da musculatura esquelética ao longo do envelhecimento ocorrem de maneira diferenciada para cada indivíduo, sendo influenciada pelos fatores intrínsecos e extrínsecos ao organismo (LEITE et al., 2012). Talvez no presente estudo, a variação dos resultados de força muscular entre grupos de idosas sedentárias, ativas e muito ativas, também recebeu influência de fatores genéticos, histórico de vida entre outros. Um programa de fortalecimento muscular específico, mediante as necessidades e particularidades de cada idoso, estimula o envelhecimento saudável, previne doenças, melhora a capacidade funcional e qualidade de vida (STRAIGHT et al., 2016).

Miranda (2014) analisou os efeitos da hidroginástica sobre o aumento de FM de membros inferiores em idosas. Os resultados obtidos demonstraram diferenças significativas no aumento da FM de membros inferiores e que a prática de hidroginástica por idosos deve ser estimulada não somente para desenvolvimento cardiorrespiratório, mas também para a melhora da FM.

A hidroginástica proporciona efeitos significativos sob a FM de membros inferiores, bem como, benefícios emocionais, aumento da sociabilidade, melhora na estabilidade postural e marcha, o que pode 
prevenir ou reduzir as quedas (GARCIA et al., 2016).

A aptidão física que é composta por capacidades físicas são essenciais para realização das atividades diárias, bem como a minimização do risco de doenças crônicas (POMPEMRAYER; GONÇALVES, 2011).

A maioria das idosas praticantes de hidroginástica apresentou NAF física classificada como "ativo" ou "muito ativo". Tal resultado não é concordante com pesquisas prévias (PÍCOLI; FIGUEIREDO; PATRIZZI, 2011; LEITE et al., 2012), nas quais observaram a diminuição progressiva no NAF após os sessenta anos de idade, sendo mais acentuada naqueles com setenta anos e mais de idade. Notoriamente, nossos achados são relevantes, visto que a atividade física em nível adequado em idosos age diretamente sobre o declínio biológico, diminuição da FM e excesso de peso corporal na velhice, além de auxiliar na manutenção da capacidade aeróbia (SARDELI; BONGANHA; CAMARA, 2014).

Contudo, como supramencionado, o NAF elevado em praticantes de hidroginástica não se correlacionou com a melhor FM em membros inferiores. Como supramencionado, as idosas muito ativas ou ativas obtiveram menores escores de FM no segmento corporal citado. Tal achado é concordante com dos Santos et al. (2013), nos quais observaram que as idosas fisicamente ativas, praticantes de hidroginástica, não obtiveram ganhos de FM de membros superiores e inferiores.

Um bom NAF é uma dos mecanismos que pode prevenir a incapacidade funcional devido os declínios fisiológicos relacionados com o envelhecimento (VIRTUOSOJÚNIOR et al., 2016).

Embora a hidroginástica seja uma modalidade que traz melhores condições de saúde para os idosos é necessário um trabalho conjunto com treinamento resistido e para membros superiores e inferiores, mediante as perdas específicas e necessidades de cada indivíduo, tratando-se de exercícios físicos complementares. A musculação promove o fortalecimento e consequentemente melhora a força muscular, minimizando as perdas próprias na musculatura, além de contribuir para a redução do peso corporal no idoso (TEIXEIRA; CÔRTE, 2011).

\section{CONCLUSÃO}

Existe associação inversa do NAF com a FM de membros inferiores de idosas praticantes de hidroginástica, indicando que idosas ativas e muito ativas possuem FM fraca/muito fraca. 


\section{REFERÊNCIAS}

CERVI, Adriane; FRANCESCHINI, Sylvia do Carmo Castro; PRIORE, Sílvia Eloiza. Análise crítica do uso do índice de massa corporal para idosos. Rev Nutr, v. 18, n. 6, p. 765-775, 2005.

COELHO, Bruna dos Santos et al.

Comparação da força e capacidade funcional entre idosos praticantes de musculação, hidroginástica e não praticantes de exercícios físicos. Rev Bras Geriatr Gerontol, v. 17, n. 3, p. 497-504, 2014.

COSTA, Sebastião da Silva; CARVALHO, Rodrigo Gustavo da Silva; GOMES, Lara Elena. Efeito de um projeto de extensão de hidroginástica em capacidades físicas e em indicadores antropométricos. Revista Brasileira de Ciência e Movimento, v. 24, n. 2, p. 110-118, 2016.

GARCIA, Joice dos Santos et al. Avaliação da autonomia funcional do idoso ativo.

Revista Ciência e Saúde On-line, v. 1, n. 1, p. 51-60, 2016.

KELLER, Karsten; ENGELHARDT, Martin. Strength and muscle mass loss with aging process. Age and strength loss. Muscles, ligaments and tendons journal, v. 3, n. 4, p. 346, 2013.

KÜMPEL, Daiana Argente et al. Obesity among elderly accompanied by the brazilian family health strategy. Texto \& Contex Enferm, v. 20, n. 3, p. 471-477, 2011.

KURA, Gustavo Graeff et al. Nível de atividade física, IMC e índices de força muscular estática entre idosas praticantes de hidroginástica e ginástica. Revista Brasileira de Ciências do Envelhecimento Humano, v. 1, n. 2, p. 30-40, 2006.

LEITE, Leni Everson de Araújo et al. Envelhecimento, estresse oxidativo e sarcopenia: uma abordagem sistêmica. Rev
Bras Geriatr Gerontol, v. 15, n. 2, p. 365-380, 2012.

MACIEL, Daniele Cristina Rodrigues; MACIEL, Luiz Henrique Rezendo. Efeitos de um treinamento de hidroginastica rerententes a melhoria no imc (indice de massa corpórea) das praticantes. Revista ENAF Science, v. 6, n. 2, p. 34-38, 2011.

MATSUDO, Sandra et al. Questinário internacional de atividade f1 sica (IPAQ): estudo de validade e reprodutibilidade no Brasil. Rev Bras Ativ Fís Saúde, v. 6, n. 2, p. 5-18, 2001.

MIRANDA, Humberto et al. PROTOCOLO GDLAM. Fitness \& Performance Journal, v. 8, n. 5, p. 378-382, 2009.

MIRANDA, Juliana Ester de. Efeitos da Hidroginástica sobre o aumento da força de membros inferiores em idosas. RBPFEXRevista Brasileira de Prescrição e Fisiologia do Exercício, v. 8, n. 48, p. 628-634, 2014.

NESI, Patrícia Pandini. A contribuição da hidroginástica na qualidade de vida de idosas. 2015.

PÍCOLI, Tatiane da Silva, FIGUEIREDO, Larissa Lomeu, PATRIZZI, Lislei Jorge. Sarcopenia e envelhecimento. Fisioter Mov, v. 24, n. 3, p. 455-462, 2011.

POMPERMAYER, Marcelo Gava; GONÇALVES, Andréa Krüger. Relação entre capacidades motoras de idosas praticantes de hidroginástica e alongamento. Estudos Interdisciplinares sobre o Envelhecimento, v. 16, n. 1, p. 473-484, 2011.

RIKLI, Roberta E. Teste de aptidão física para idosos. Manole, 2008.

SANTOS, Rafaela G. dos et al. Força de membros inferiores como indicador de incapacidade funcional em idosos. Motriz, v. 19, n. 3, p. 35-42, 2013. 
SARDELI, Amanda Veiga; BONGANHA, Valéria, AMARA, Fabiano Marques. Relação entre nível de atividade física e qualidade de vida no envelhecimento. Estudos Interdisciplinares sobre o Envelhecimento, v. 19, n. 2, p. 485-499, 2014.

SGARIBOLDI, Dayla et al. Influência da idade, das características antropométricas e da distribuição de gordura corporal na mobilidade torácica de mulheres. Fisioterapia e Pesquisa, v. 22, n. 4, p. 342-347, 2015.

SILVA, Adriana; DAL PRÁ, Keli Regina. Envelhecimento populacional no Brasil: elementos para pensar o lugar das familias na proteção dos idosos. Argumentum, v. 6, n. 1, p. 99-115, 2014.

SILVA, Valter et al. Acompanhamento de curto prazo de indicadores de obesidade em idosas obesas frágeis: efeitos da intervenção com hidroginástica. Revista Brasileira de Prescrição e Fisiologia do Exercício, v. 6, n. 36, p. 1-7, 2012.

SOUTO, Simone Valéria Dias et al. Body image in adult vs. middle-aged and elderly women practitioners and non-practitioners of hydro gymnastics. Motricidade, v. 12, n. 1, p. 53-59, 2016.

SOUZA JÚNIOR, Sálvio Santos et al. Força de membros superiores e inferiores de idosas praticantes e não praticantes de ginástica funcional. Saúde, v. 41, n. 1, p. 255-262, 2015.

SOUZA, Raphaela et al. Avaliação antropométrica em idosos: estimativas de peso e altura e concordância entre classificações de IMC. Rev Bras Geriatr Gerontol, v. 16, n. 1, p. 81-90, 2013.

STRAIGHT, Chad R. et al. Effects of resistance training on lower-extremity muscle power in middle-aged and older adults: a systematic review and meta-analysis of randomized controlled trials. Sports Med, v. 46, n. 3, p. 353-364, 2016.
TEIXEIRA, Tatiane Gomes; CÔRTE, Beltrina. Conhecimento sobre benefícios associados à prática de musculação por idosos físicamente ativos. Geriatric \& Gerontol, v. 5, n. 4, p. 233-241, 2011.

VEDANA, Tatiana Andrade et al. Influência da hidroginástica sobre a composição corporal, aspectos cardiovasculares, hematológicos, função pulmonar e aptidão física de adultos e idosos. Brazilian Journal of Biomotricity, v. 5, n. 2, p. 65-79, 2011.

VIEIRA, Elciana de Paiva Lima et al. Efeito crônico do treinamento de flexibilidade sobre o controle postural de idosas praticantes de hidroginástica. 2014.

VIRTUOSO- JÚNIOR, J. S. et al. Fatores associados à incapacidade funcional em idosos brasileiros. Revista Andaluza de Medicina del Deporte, n. 129, p. 1-7, 2016.

ZUCOLO, André Chang et al. Efeitos da hidroginástica sobre variáveis morfofuncionais de indivíduos de meia idade e idosos. RBPFEX-Revista Brasileira de Prescrição e Fisiologia do Exercício, v. 9, n. 54, p. 457-462, 2016. 